\title{
Optimising QoE Distribution for Video Applications through LTE-WiFi Interworking
}

\author{
Mirghiasaldin Seyedebrahimi, Xiao-Hong Peng \\ School of Engineering and Applied Science \\ Aston University \\ Birmingham B4 7ET, UK. \\ \{seyedebm, x-h.peng\}@aston.ac.uk
}

\begin{abstract}
Mobile WiFi devices are becoming increasingly popular in non-seamless and user-controlled mobile traffic offloading alongside the standard WiFi hotspots. Unlike the operator-controlled hotspots, a mobile WiFi device relies on the capacity of the macro-cell for the data rate allocated to it. This type of devices can help offloading data traffic from the macro-cell base station and serve the end users within a closer range, but will change the pattern of resource distributions operated by the base station. We propose a resource allocation scheme that aims to optimize user quality of experience (QoE) when accessing video services in the environment where traffic offloading is taking place through interworking between a mobile communication system and low range wireless LANs. In this scheme, a rate redistribution algorithm is derived to perform scheduling which is controlled by a no-reference quality assessment metric in order to achieve the desired trade-offs between efficiency and fairness. We show the performance of this algorithm in terms of the distribution of the allocated data rates throughout the macro-cell investigated and the service coverage offered by the WiFi access point.
\end{abstract}

Keywords-Quality of Experience; interworking; WiFi offloading; LTE; resource allocation; video streaming

\section{INTRODUCTION}

There has been significant growth in the penetration rate of smart devices and overall mobile traffic driven by increasing video and cloud usages. The new generation mobile systems $(4 \mathrm{G} / 5 \mathrm{G})$ is required to provide a ubiquitous high Quality of Experience (QoE) to mobile users while meeting the capacity needs of the operators and content providers [1]. This situation has been echoed in the latest standardization of the advanced Long Term Evolution (3GPP-LTE-A) [2]. The combined technological solutions such as Small Cells, WiFi offloading, Relays and Self-Organizing Network (SON) have been considered within the mobile communication system to overcome its capacity limits. These solutions would be especially attractive if they do not require a complex upgrade of the existing network architecture.

A proximity service, such as offloading through interworking with $\mathrm{WiFi}$ hotspots (based on the IEEE 802.11u wireless LAN (WLAN) [3]), acts as a congestion reliever in LTE networks [4]. This service can improves end-user QoE through proper distribution of network capacity and regulate the radio power consumption. Higher throughput and lower cost per bit are expected to be achieved by using small cells or interworking with previously established WLAN hotspots at a low cost.
The current standardization on data offloading deals mainly with the IP routing mechanism, breakout point issues and the way to redirect user data from LTE to a different connection point. The proposed architecture of the IP Flow Mobility (IFOM) in relation with LTE's Evolved Packet Core (EPC) [5] is an example of these efforts. However, there are still many issues open for further research on traffic offloading in LTE such as the capacity distribution pattern, effects of the scheduling policy, trade-offs between fairness and efficiency, and the backbone connection strategy for hotspots. In this work we will examine these aspects in the context of data offloading in LTE for video streaming traffic.

We propose an offloading solution for video streaming services with the optimization of QoE distribution across the network through the interworking between LTE base station (or eNodeB) and WiFi hotspots. Pause Intensity (PI), as a no-reference and packet based metric for QoE assessment, is applied to derive the resource allocation strategy. The offloading performance of this solution will be discussed in detail later.

We will examine two different offloading methods, one through the hotspot which has an independent backbone connection and the other using a WiFi access point as a user of a macro-cell base station. The former type of the WiFi access points are defined in 3GPP standards as Interworking WLAN (I-WLAN) [4]. The latter is commercially known as Mobile WiFi device (MiFi) which resembles a small cell or femtocell with an unlicensed radio spectrum and a macro-cell dependant backbone connection. The performances of these two methods will be analysed and compared.

$\mathrm{MiFi}$ is regarded as a standalone LTE-WiFi device to provide connections with improved reception quality. For device-to-device (D2D) communications, tablets and smartphones can also be used to relay the $3 \mathrm{G} / 4 \mathrm{G}$ connection using their $\mathrm{WiFi}$ port to adjacent users. However, these personal devices have a lower reception quality compared to a standalone MiFi device. These two options are available for the user equipment (i.e. UE) to choose for connecting the backbone network, but the resource allocation mechanism at the eNodeB needs additional intelligence to ensure the overall network performance to be optimal, in terms of the capacity utilization and trade-off between efficiency and fairness. This issue is address by a scheme proposed in this work.

The rest of the paper is organized as follows. The background and related works are explained in Section II. The proposed rate redistribution and QoE optimization schemes for the LTE-WiFi interworking system are 
presented in Section III. Section IV provides simulation results and detailed analysis on the performance of the proposed solution. Finally, the conclusion is given in Section V.

\section{BACKGROUND AND RELATED WORKS}

The network capacity or the total offered data rate supported by the new generation mobile communication technologies has not been fully utilized across the area covered by a macro-cell base station. Furthermore, any promised capacity is highly conditional and depends on the reception quality of the user device, the distribution of users in the cell and, most importantly, the resource allocation and scheduling policies. In fact, most of the users at the edge of the cell will not benefit from the improved capacity of the network if they rely purely on their direct connection to the macro-cell base station. The 3GPP standardization has considered WLAN traffic offloading as a solution for maintaining the performance of the service at its required level [4]. This will be the case whenever users are better off in receiving their service through a WiFi hotspot than a direct macro-cell connection. .

Although the interworking between WLAN and LTE is a relatively new subject, the main concept of offloading through interworking between different types of the networks (due to the heterogeneity of the network) has been associated with other techniques such as load balancing [6], [7], resource management [8] and congestion control [9].

The different aspects of the WLAN offloading mechanism in the context of the new generations of the mobile communication systems (e.g. LTE-Advanced) are currently under development. An offloading process through WiFi hotspot can be seamless if without service interruption or non-seamless otherwise [10]. Offloading may require the initiation and signaling of the user equipment and can also happen even without the intervention of user device (e.g. client and network based IP mobility in IFOM [5]). The performance of the standardized traffic offloading mechanism is also being investigated together with small-cell and D2D communication technologies [11]-[13]. In collaboration

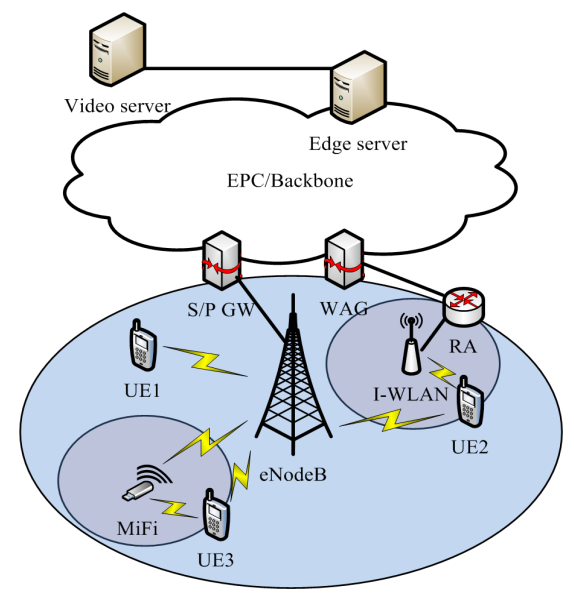

Figure 1. Different types of video streaming service provided through a direct connection to the base station (UE1), offloading using an independent WiFi access point (UE2 through I-WLAN), or offloading using a mobile WiFi (UE3 through MiFi). with protocols at higher layers, the traffic offloading mechanism has been employed to improve the performance at the application layer in a cross-layer paradigm [14].

All the previous works mentioned above are based on the conventional interworking between offloading hotspots and the mobile system while the new emerging standalone and user-controlled mobile WiFi devices are not considered in this scenario. Traffic offloading through a mobile WiFi device reshapes the distribution of the available capacity (in terms of the allocated data rate to users) throughout the cell, but this still relies on the capacity of the mobile base station. In the following sections, this issue is addressed by a proposed resource allocation method including the rate redistribution algorithm and its performance evaluation.

\section{MODEL OF RATE ALLOCATION}

Fig. 1 shows a macro-cell base station (known as eNodeB in LTE) where different traffic offloading mechanisms are depicted. In this scenario, user equipment 1(UE1) has a normal connection to the server directly through the macro-cell base station. UE2, however, receives the service through offloading via a WiFi access point which has a backbone connection independent from macro-cell. In this case, a router (RA) and a wireless access gateway (WAG) are usually used to provide the backbone connection for enabling IP mobility and interworking with LTE-EPC, which is the standard form of the offloading mechanism and known as I-WLAN. For UE3, the connected WiFi access point is a portable/mobile WiFi device called Mobile WiFi or MiFi. MiFi is connected to the backbone through the LTE base station while UE3 will remains to be the user of the macro-cell base station. In contrast to the I-WLAN, a MiFi is not under the control of network operators and can serve a limited number of mobile or fixed devices for partial or full offloading purposes.

Based on the environment described above, the quality assessment metric employed, conditions for resource allocation in LTE and the rate redistribution algorithm will be discussed in the rest of this section, in the context of video streaming services. The performance of the proposed algorithm will be analyzed later in Section IV.

\section{A. QoE Metric}

Pause Intensity, PI, is a metric for quality assessment which quantifies the video streaming playback discontinuity by characterizing the playout buffer behavior. PI considers both pause duration and pause frequency and is also a function of the network throughput $\eta$ and video encoding/decoding rate $\lambda[15]$, i.e.:

$$
P I=1-\frac{\eta}{\lambda}
$$

The video encoding rate is regarded as the service level or data allocation rate required by a user. In a streaming scenario $\eta$ is normally less than or equal to $\lambda$ and $0 \leq \mathrm{PI} \leq 1$. 
It has been shown that PI is closely correlated with the subjective quality evaluation metric, MOS (Mean Opinion Score) and independent from the video content provided [15], as shown in Fig.2. Therefore, PI can be used to estimate the level of user's QoE in order for network operators and service providers to allocate proper resources to end users in 3GPP-LTE networks.

In a macro-cell that serves a large number of mobile devices, it is essential to maintain a good balance between the efficiency and fairness in delivering data to users with different conditions. As an extension to the original PI metric, the high-order PI, i.e.: $P I^{\alpha}(0 \leq \alpha)$, can be introduced to act as a weighting coefficient for rate allocation with QoE awareness. The detailed descriptions of the highorder PI and its application in the rate redistribution algorithm are given in Subsection III- $C$ and Section IV, respectively.

\section{B. Resource allocation for LTE}

Each user of the LTE base station (eNodeB) provides an estimation of its channel status across the available resource blocks, $\mathrm{N}_{\mathrm{RB}}$, based on the signal-to-noise ratio, such as

$$
S N R \in\left\{S N R_{\min }, \ldots, S N R_{\max }\right\}^{1 \times N_{R B}}
$$

A CQI (Channel Quality Indication) feedback will be generated based on the above e given the capability of the client's device, which is defined as

$$
C Q I \in\left\{1,2, \ldots, C Q I_{\max }\right\}^{1 \times N_{R B}}
$$

The value of CQI can be a result of a linear fitting of SINR value(s) or searching through a lookup table similar to the example shown in Table I. These values reflect the capability of the user's device with regard to different modulation and channel code rates (MCS) to achieve a minimum acceptable error rate. CQI suggests a range of modulation and code rates for which at least a $90 \%$ successful rate will be achievable at the receiver. Given the selected modulation order and channel code rate (based on the CQI values) and the allocated resources, $r_{k}$, the total allocated data rate to user $k\left(k=1\right.$ to $\left.N_{U E}\right)$ in the $i^{\text {th }}$

TABLE I. LINK ADAPTATION AND MODULATION SCHEME

\begin{tabular}{|c|c|c|c|}
\hline SINR & CQI & Modulation Order & Code Rate \\
\hline$\leq-6.934$ & $1(*)$ & 2 & 0.1523 \\
\hline-5.147 & 2 & 2 & 0.2344 \\
\hline-3.180 & 3 & 2 & 0.3770 \\
\hline-1.254 & 4 & 2 & 0.6016 \\
\hline 0.7610 & 5 & 2 & 0.8770 \\
\hline 2.700 & 6 & 2 & 1.1758 \\
\hline 4.697 & 7 & 4 & 1.4766 \\
\hline 6.528 & 8 & 4 & 1.9141 \\
\hline 8.576 & 9 & 4 & 2.4063 \\
\hline 10.37 & 10 & 6 & 2.7305 \\
\hline 12.30 & 11 & 6 & 3.3223 \\
\hline 14.18 & 12 & 6 & 3.9023 \\
\hline 15.89 & 13 & 6 & 4.5234 \\
\hline 17.82 & 14 & 6 & 5.1152 \\
\hline$\geq 19.83$ & 15 & 6 & 5.5547 \\
\hline
\end{tabular}

* Users with a SINR lower than a device related threshold will not be scheduled for resource allocation.

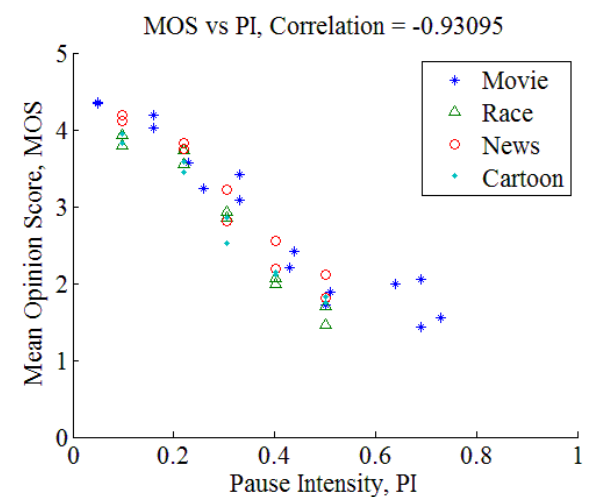

Figure 2. Correlation between MOS and PI based on subjective tests.

round of the allocation, $R_{k}^{i}$, can be calculated as:

$$
\left\{\begin{array}{c}
R_{k}^{i}=C_{k}^{T} \cdot r_{k} \\
C_{k} \in \mathbb{R}_{>0}^{N_{R B} \times 1}, r_{k} \in\{0,1\}^{N_{R B} \times 1}
\end{array}\right.
$$

where $r_{k}$ is the vector of the allocation and $C_{k}$ is the vector of the achievable capacities in the resource blocks for user $k$, given the corresponding CQI values (i.e. $\mathrm{C}_{\mathrm{k}}=\mathrm{f}(\mathrm{CQI}(\mathrm{SINR}))$ as illustrated in Table I). Formula (4) will be used to model the overall capacity of the system in the next subsection.

\section{Rate Redistribution Algorithm}

Based on the structure of the last-mile wireless connection for a video streaming service (shown in Fig. 1), user can either be served by the LTE macro-cell base station (eNodeB), or through a WiFi hotspot whenever an offloading mechanism is in place. Each hotspot can be connected to the backbone through its broadband connection which is independent from the mobile base station. It can also be connected to the backbone as a user of the mobile base station or as a MiFi device which has been explained previously.

An independent broadband connection to the backbone for $\mathrm{WiFi}$ hotspots will increase the overall capacity in terms of the allocated data rate in the cell. But a hotspot which is connected to the base station doesn't increase the total available resources in the cell. The capacity is increased because users connected to a MiFi have better reception quality and can help improve the resource distribution with MiFi's own capacity. In this case, the link adaptation and resource utilization will be improved for those users as long as the MiFi device provides a better reception compared to eNodeB for mobile users. Essentially, the locations of the offloading hotspots and the policy adopted by the scheduler in the macro-cell base station are the main factors in reshaping the distribution of the data rates to the users.

Consider a macro-cell with an LTE base station, $\mathrm{N}_{\mathrm{UE}}$ mobile users, and $\mathrm{N}_{\mathrm{AP}} \mathrm{WiFi}$ hotspots. The total provided capacity in the cell will be a combination of the allocated data rates to the users by the main base station and the surrounding hotspots, i.e.:

$$
R_{t}=R_{e N B}+\sum_{k=1}^{N_{A P}} R_{\mathrm{AP}_{k}}
$$


where $R_{e N B}$ and $R_{A P}$ are the allocated data rates to the users by the base station and WiFi hotspots, respectively. Scheduling and link adaptation functions in the base station serve all users in the cell except for those who are under the coverage of the I-WLAN hotspots. Those users are assumed to be served entirely through a sufficient WiFi broadband connection. As a result, the $R_{\mathrm{APk}}$ will be the summation of all the offloaded traffic of the $k$-th IWLAN. However, in the case of MiFi, the Head-of-Line packets (HOL) related to the users under its coverage will be scheduled by eNodeB as a part of the MiFi's data.

An efficiency-oriented scheduler in eNodeB (e.g. BestCQI) will maximize the total allocated data rate, $R_{t}$, while a fairness-oriented scheduling policy will provide a flat data rate allocation among the users. The efficiency and fairness of the system will be evaluated in this work based on the total allocated data rates and the Jain's index of the allocated data rates, respectively.

By using the high-order PI metric defined in Subsection III- $A$ and the user capacity in (4), we have

$$
\left\{\begin{array}{c}
x^{*}=\arg x \max f^{T} x \\
f \in \mathbb{R}^{N_{U E} \times 1}, \quad f_{k}=P I_{k}{ }^{\alpha} \cdot \overline{c_{k}} \\
x \in \mathbb{Z}_{\geq 0}^{N_{U E} \times 1}, \quad x_{k} \leq N_{R B}
\end{array}\right.
$$

where $f_{k}$ is regarded as a utility function and combines the effect of the user experienced quality, represented by PI, with its average achievable data rate, $\overline{c_{k}}$ (i.e. user efficiency). The integer value $x^{*} \geq 0$ is the number of the allocated resources to user $k$ (i.e. $x^{*}{ }_{k}=\left\|r_{k}\right\|_{l}$ ) during the scheduling process, based on which the total allocated data rate can be expressed as:

$$
R_{e N B}=\bar{C} \cdot x^{T}, \quad \bar{C}=\left[\overline{c_{1}}, \ldots, \overline{c_{N_{U E}}}\right] \in \mathbb{R}^{1 \times N_{U E}}
$$

where $C$ is the vector of the achievable data rates for each user if a single resource block is allocated to that user. $c_{k}$ is a function of the SINR and CQI at the receiving side and is assumed to be the same for all resource blocks (i.e. throughout the available bandwidth). Since a MiFi device mediates between its users and the mobile base station, the users will benefit from the higher SINR and subsequent higher $c_{k}$ provided by the MiFi device.

Parameter $\alpha(\alpha \geq 0)$ defines the degree of the influence of QoE over the scheduling process. Since $0 \leq \mathrm{PI} \leq 1$, the value of $c_{k}$ dominates the utility function, $f_{k}$, for small values of $\alpha$. Consequently, it leads to a more efficient scheduling process. In contrast, with large $\alpha$ the network efficiency or throughput will be reduced but fairness among the users will be improved as an attempt to achieve the required user QoE. More discussions on the QoE issue and performance results will be presented later in Section IV. An implementation algorithm for the optimization model given in (6) can be expressed as

$$
\left\{\begin{array}{l}
k^{*}=\arg _{k} \max u_{k} \\
u_{k}=P I^{\alpha} \cdot c_{k}
\end{array}\right.
$$

where $u_{k}$ as a priority function allows the scheduler to choose user $k$ with the dominant value of $u_{k}$ in each round
TABLE II. Simulation SetuP

\begin{tabular}{lc}
\hline Parameter & value \\
\hline $\begin{array}{l}\text { No. of Cells } \\
\text { Inter-site distance }\end{array}$ & $\begin{array}{c}\text { (with the first tier interference) } \\
\text { mean }=00 \text {, deviation }=8\end{array}$ \\
Shadowing effect & $\begin{array}{c}\text { correlation }=0.5 \\
\text { decorrelation distance }=25 \mathrm{~m}, \text { inter-site } \\
\text { Channel model }\end{array}$ \\
Bandwidth & PedA, speed $=3 \mathrm{~km} / \mathrm{h}$ \\
No. of RBs (per TimeSlot) & $20 \mathrm{MHz}$ \\
Subcarrier & 100 \\
Range of average SINR & $15 \mathrm{KHz}$ \\
Average video code rate & $-6 \sim 18 \mathrm{~dB}(\mathrm{CQI}=1 \sim 15)$ \\
No of Users & $790 \mathrm{kbps}$ \\
Each scheduling round & 130 (in each simulation run) \\
Simulation time & One TTI $=1 \mathrm{~ms}$ \\
Video stream model & $10000 * \mathrm{TTI}(10 \mathrm{~s})$ \\
WiFi standard/coverage & Truncated Pareto for packet size and \\
MiFi device antenna gain & inter-arrival time \\
\hline
\end{tabular}

of the scheduling. This reduces the complexity of the implementation and processing requirements compared to (6), and its performance results closely match the model. In Section IV, the performance of the proposed implementation algorithm in (8) will be compared with the analytical model in (6) for different interworking scenarios.

\section{SIMULATION RESULTS AND ANALYSIS}

\section{A. Simulation setup}

Table II shows the settings of the simulator developed in Matlab to examine the proposed QoE-driven rate redistribution algorithm for interworking between $\mathrm{WiFi}$ and LTE. The user data are the video stream packets generated using a truncated Pareto model (for packet interarrival-time and size). No background traffic is considered. Video code rate has been set to a standard video streaming quality of $790 \mathrm{kbps}$ (e.g. as used in BBCiPlayer). User Head-of-Line packets (HOL) are scheduled in a timely manner with no packet drop due to the delay in the scheduler at eNodeB. The mapping between SINR and $\mathrm{CQI}$ for lower layer function settings such as the modulation order and channel code rate is given in Table I.

The same video code rate is used for users with different SINRs in the range of the defined CQI for LTE (i.e. 1 15). Users are distributed in one cell and the interference is considered from the first tier neighboring cells. A pedestrian user model of fading and the shadowing effect (inter/intra-cell spatial correlation) are taken into account as well. The subjective quality measurements of the service (MOS) are estimated through the fitting of the stable value of PI given the subjective test results in Fig. 2. Fig. 3 shows the received power, geometric SINR and CQI distribution of the simulated network.

\section{B. Results and Analysis}

All the results presented in this section are obtained through five independent runs of the simulation for 100 randomly located users and 30 users with fixed locations. Users with fixed locations are capable of acting as a LTE mobile user and/or WiFi offloading device. Fig. 4(a) 


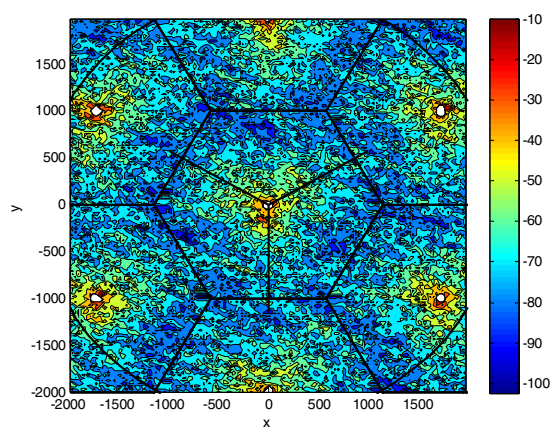

(a) Received power $[\mathrm{dBm}]$

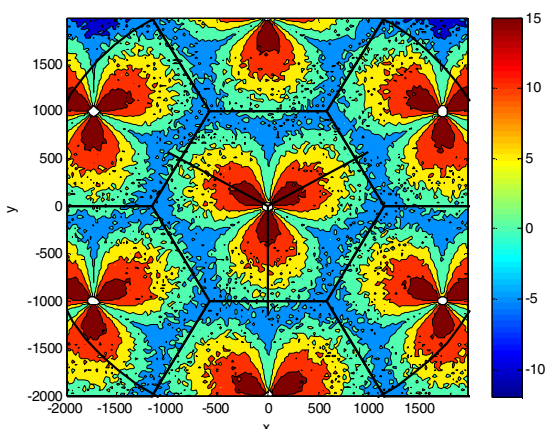

(b) SINR geometry $[\mathrm{dB}]$.

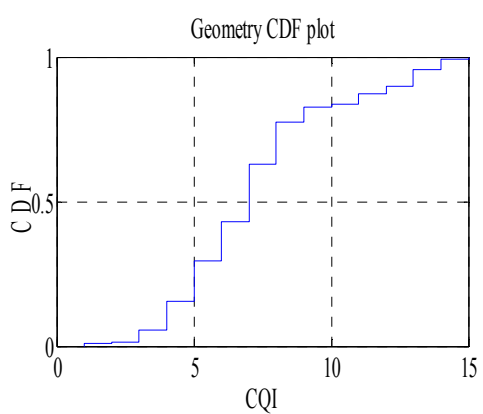

(c) CQI values distribution.

Figure 3. The geometric properties of the network concerned.

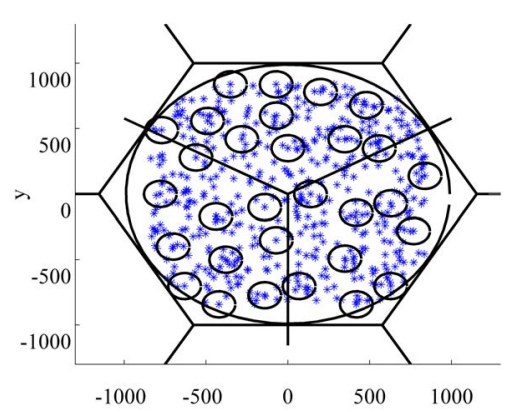

(a) User locations and WiFi access point coverage

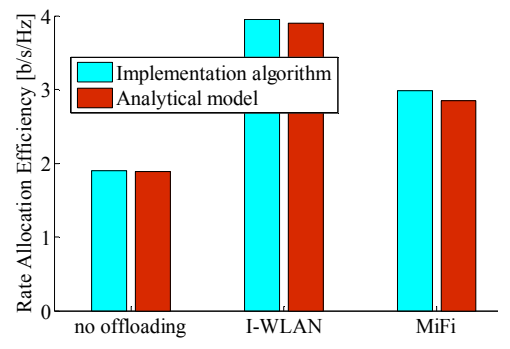

(b) Rate allocation efficiency in different scenarios

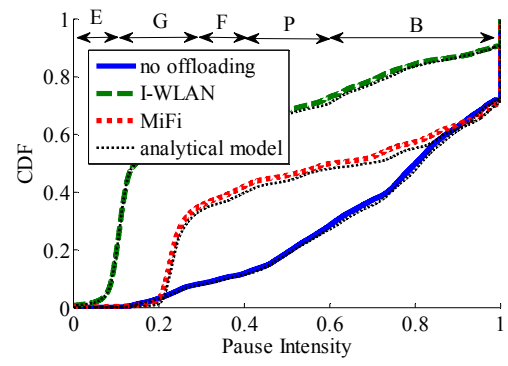

(c) Achieved QoE based on PI values

Figure 4. Comparisons between different LTE-WiFi interworking scenarios for the proposed analytical model and the implemented algorithm in (6) and (8). The letters E, G, F, P and B in (c) represent Excellent, Good, Fair, Poor and Bad qualities based on the corresponding MOS values.

shows a total of 500 examined locations and the coverage of the remaining 30 users when they act as WiFi access points. Fig. 4(b) shows the total provided capacity, in terms of the allocated data rate, with and without WiFi interworking. In Fig. 4(c) the distribution of the achieved PI (for QoE) is depicted. The results of the implementation algorithm in (8) and those for the analytical model in (6) are shown to be closely matched in both Fig. 4(b) \& 4(c).

The WiFi hotspots with the independent backbone connection (i.e. I-WLAN) bring in extra capacity which contributes to the increase of the total capacity in the cell. In contrast, the standalone MiFi devices which rely on the capacity of the base station do not increase the available resources in the cell. However, due to their better reception quality compared to the neighboring mobile users, their link adaptation schemes can provide a higher data rate for users under their coverage. The results in Fig. 4 show that the macro-cell will benefit from interworking regardless of the type of the $\mathrm{WiFi}$ backbone connections. However, as it will be shown later in this section, this will not always be the case for any scheduling policy used by the base station.

Fig. 5 reveals how the interworking mechanisms discussed above restructure the distribution of the allocated data rate in the cell, illustrated by the twodimensional geometric maps. The color bar on the right of Fig. 5(a) shows the correspondence between the color used in the map and the allocated rate. The distribution shown in Fig. 5(a), where no offloading mechanism is applied, has a similar shape to the geometric SINR in the cell shown in Fig. 3(b). In the case of I-WLAN offloading shown in Fig. 5(b), the extra capacity provided by the independent backbone connection of the WiFi hotspots improves the capacity of the cell. Furthermore, Fig. 5(b) demonstrates how the locations of WiFi hotspots influence the redistribution of the allocated data rate, compared to the case without offloading given in Fig. 5(a).

Fig. 5(c)-(e) show the redistributed allocated data rate for the $\mathrm{MiFi}$ interworking scenario. The scheduling algorithm given in (8) is used by the macro-cell base station with $\alpha=0.1,1$ and 10 , respectively. It can be seen that small $\alpha$ tends to enable a more efficient scheduling policy, whilst large $\alpha$ will result in a more fair scheduling policy. These results also show how the MiFi offloading performance is affected by the scheduling policy, due to the dependency of MiFi over the mobile base station.

From Table III, we can notice the trade-off between the fairness and efficiency of the system. As it is shown, the parameters representing both fairness and efficiency are proportional to the value of the scheduler parameter $\alpha$, but with opposite trends to each other. In the same table the achieved user QoE is also exhibited through the comparison of the $50^{\text {th }}$ percentile of the achieved MOS values in each case.

The contrast among the allocated rates, in terms of the difference in color displayed in the rate distribution maps in Fig. 5, also indicates the levels of fairness and efficiency. The parameter $\alpha$ can be used to regulate the level of contrast in order to control the balance between fairness and efficiency performances. For example, increasing $\alpha$ will reduce the contrast of the rate redistribution map and make the system more fair in terms 


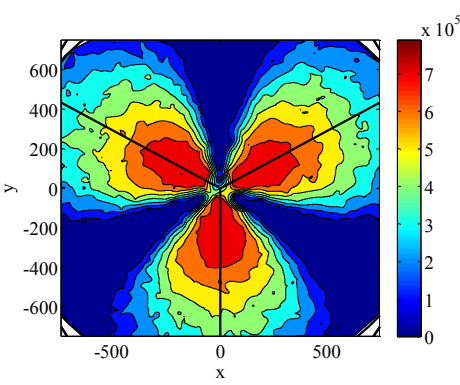

(a) without traffic offloading

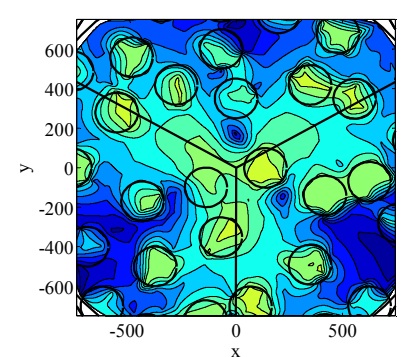

(e) MiFi and a fair scheduler $(\alpha=10)$

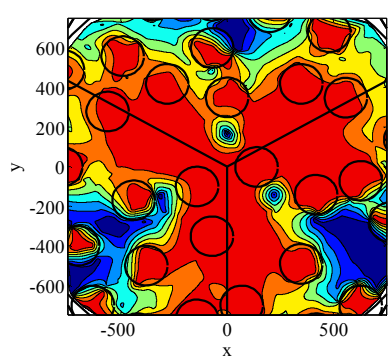

(b) I-WLAN hotspots

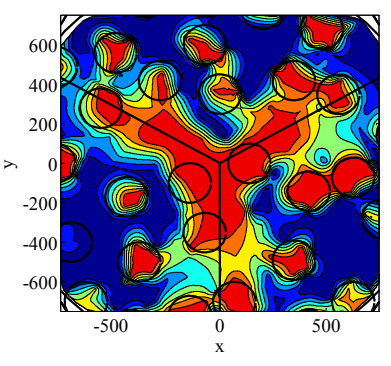

(c) MiFi and an efficient scheduler $(\alpha=0.1)$

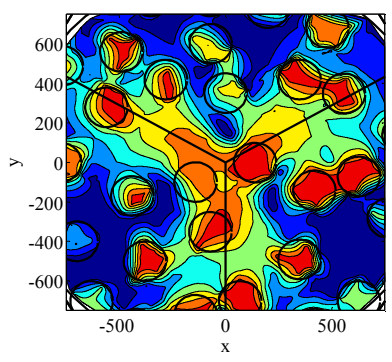

(d) MiFi and an intermediate scheduler $(\alpha=1)$

TABLE III. MiFI PERFormanCE

\begin{tabular}{|c|c|c|c|}
\hline $\begin{array}{c}\text { Scheduler } \\
\text { parameter } \\
(\alpha)\end{array}$ & $\begin{array}{c}\text { Achieved } \\
\text { Efficiency } \\
(\mathrm{b} / \mathrm{s} / \mathrm{Hz})\end{array}$ & $\begin{array}{c}\text { Achieved } \\
\text { fairness } \\
(\mathrm{J}-\text {-index })\end{array}$ & $\begin{array}{c}\text { Achieved MOS } \\
\text { Quality } \\
\left(50^{\text {Th }} \text { percentile) }\right.\end{array}$ \\
\hline 0.1 & 3.2 & 0.63 & $\begin{array}{c}50 \% \text { above } 3.2 \\
\text { (Avg. Good) }\end{array}$ \\
\hline 1 & 2.9 & 0.68 & $\begin{array}{c}50 \% \text { above } 2.1 \\
\text { (Avg. Fair) }\end{array}$ \\
\hline 10 & 2.16 & 0.84 & $\begin{array}{c}50 \% \text { above } 1.6 \\
\text { (Avg. Poor) }\end{array}$ \\
\hline
\end{tabular}

Figure 5. Comparison between the allocated rate distribution in the cell for different interworking scenarios and macro-cell scheduling policies.

of resource allocation. However, a very fair scheduler like the one shown in Fig. 5(e) would decrease the satisfaction level of users as many premier users are allocated unacceptably low rates and the network efficiency measured by the throughput will be affected as a result. The opposite is also true if a very efficient scheduler is used when $\alpha$ is small.

\section{CONCLUSION}

A rate redistribution scheme for traffic offloading through mobile WiFi devices with LTE backbone connection (MiFi) has been investigated in this paper. The performance of various interworking scenarios, in terms of the allocated data rate per user, has been evaluated within a QoE-driven and parametric scheduling algorithm. The distribution of the allocated data rate for a video streaming service in a macro-cell can be controlled to achieve the desired trade-off between fairness and efficiency of the mobile network. If LTE-WiFi interworking uses a fair scheduling policy to determine the rate distribution based on the original geometric dependency in the cell, it could reduce the efficiency of the system and degrade the level of perceived quality in the cell. The rate redistribution algorithm proposed in this work is able to optimize the QoE performance of WiFi offloading in the LTE network by applying the high-order PI metric, as described above, to achieve the efficiency-fairness balance required.

\section{REFERENCES}

[1] Cisco Systems, Cisco Visual Networking Index: Global Mobile Data Traffic Forecast Update, 2013-2018, Cisco Systems, Inc., Feb. 2014.

[2] 3GPP LTE and LTE-Advanced Technology, http://www.3gpp.org/ftp/Specs/html-info/36-series.htm

[3] IEEE Std 802.11u-2011 - Part 11: Wireless LAN Medium Access Control and Physical Layer Specifications: Amendment 9, 2011.
[4] 3GPP TS 23.234, "3GPP System to Wireless Local Area Network (WLAN) interworking; System Description,” Rel. 11, Sep. 2012.

[5] 3GPP TS 23.261, "IP flow mobility and seamless Wireless Local Area Network (WLAN) offload; Stage 2,” Rel. 11, Sep. 2012.

[6] A. Awada, B. Wegmann, I. Vierin, and A. Klein, "A game-theoretic approach to load balancing in cellular radio networks," Proc. IEEE Int. Conf. on Personal Indoor and Mobile Radio Communications, Sept. 2010

[7] J. Liu, Y. Kawamoto, H. Nishiyama, N. Kato, and N. Kadowaki, "Device-to-device communications achieve efficient load balancing in LTE-advanced networks," IEEE Wireless Communications, vol.21, no.2, pp.57,65, April 2014.

[8] X. Zhuo, W. Gao, G. Cao, and Y, Dai, "Win-Coupon: An incentive framework for 3G traffic offloading," Proc. IEEE Int. Conf. on Network Protocols, Oct. 2011.

[9] M. Radenkovic, and A. Grundy, "Framework for utility driven congestion control in delay tolerant opportunistic networks," Proc. Int. Conf. on Wireless Communications and Mobile Computing, July 2011

[10] W. Yoon, and B. Jang, "Enhanced non-seamless offload for LTE and WLAN networks," IEEE Communications Letters, vol.17, no.10, pp.1960-1963, October 2013

[11] L. Hu, C. Coletti, N. Huan, IZ. Kovács, B. Vejlgaard, R. Irmer, and N. Scully, "Realistic indoor Wi-Fi and femto deployment study as the offloading solution to LTE macro networks," Proc. IEEE Vehicular Technology Conf. (VTC Fall), Sept. 2012.

[12] A. Pyattaev, K. Johnsson, S. Andreev, and Y. Koucheryavy, "3GPP LTE traffic offloading onto WiFi Direct," Proc. IEEE Wireless Communications and Networking Conference Workshops, April 2013.

[13] M. Simsek, M. Bennis, M. Debbah, and A. Czylwik, "Rethinking offload: How to intelligently combine WiFi and small cells?," Proc. IEEE Int. Conf. on Communications, June 2013.

[14] S. Chen, Z. Yuan, and G.-M. Muntean, "A traffic burstiness-based offload scheme for energy efficiency deliveries in heterogeneous wireless networks," Proc. IEEE Globecom Workshops, Dec. 2013.

[15] M. Seyedebrahimi, C. Bailey, and X.-H. Peng, "Model and performance of a no-reference quality assessment metric for video streaming," IEEE Trans. Circuits and Systems for Video Technology, vol.23, no.12, pp.2034-2043, Dec. 2013. 